\title{
Anesthesia for Advanced Endoscopic Procedures
}

\author{
Basavana Goudra' and Monica Saumoy ${ }^{2}$ \\ ${ }^{1}$ Department of Anesthesiology and Critical Care Medicine, Hospital of the University of Pennsylvania, Philadelphia, ${ }^{2}$ Division of \\ Gastroenterology, University of Pennsylvania Perelman School of Medicine, Philadelphia, USA
}

The gastrointestinal endoscopy paradigm is rapidly changing, and technological advancements are largely responsible. In tandem, anesthesia providers are adapting to the changing needs and demands. The challenges are unique. Complications arising from the procedures are both routine, such as aspiration and hypoxia, and procedure specific, such as bleeding, pneumothorax, pneumopericardium, and pneumoperitoneum. It is crucial for the anesthesia provider to have a good understanding of the techniques employed by the endoscopist. A higher index of suspicion is also essential to diagnose and appropriately manage many of the complications. In this review, an effort is made to discuss both procedural aspects and anesthesia challenges. We hope that both endoscopists and anesthesia providers will benefit from this review. Clin Endosc 2022;55:1-7

Key Words: Anesthesia; Endobariatrics; Endoscopy; Myotomy

\section{INTRODUCTION}

Until recently, endoscopic ultrasound (EUS) and endoscopic retrograde cholangiopancreatography (ERCP) constituted the majority of advanced gastrointestinal (GI) endoscopic procedures. Although this is still the case in many centers, the last decade has seen a phenomenal increase in many other procedures, aided by advancements in technology and devices. Conditions such as morbid obesity, achalasia, and superficial cancerous and noncancerous mucosal lesions of the GI tract are regularly treated endoscopically. Some of the procedures include endoscopic submucosal dissection (ESD), endoscopic sleeve gastroplasty (ESG), and peroral endoscopic myotomy (POEM). These advanced procedures present unique challenges for anesthesia providers. While hypoxemia and pulmonary aspiration continue to test the skill and preparedness

\footnotetext{
Received: September 14, 2021 Revised: October 17, 2021

Accepted: October 18, 2021

Correspondence: Basavana Goudra

Department of Anesthesiology and Critical Care Medicine, Hospital of the University of Pennsylvania, 680 Dulles, 3400 Spruce Street, Philadelphia, PA 19104 USA

Tel: +1-215-279-4321, Fax: +1-215-615-5517, E-mail: goudrab@uphs.upenn.edu ORCID: https://orcid.org/0000-0002-5146-0424
}

(c) This is an Open Access article distributed under the terms of the Creative Commons Attribution Non-Commercial License (http://creativecommons.org/ licenses/by-nc/3.0) which permits unrestricted non-commercial use, distribution, and reproduction in any medium, provided the original work is properly cited. of the anesthesia provider, complications such as venous air embolus, pneumomediastinum, pneumothorax, subcutaneous emphysema, life-threatening bleeding, and perforation of the viscus pose additional problems. Anesthesia providers are expected to provide general anesthesia in an endoscopy suite and be prepared to address issues such as hypothermia and hypercarbia in an environment where little expert help is available. In short, the topic of anesthesia for advanced endoscopic procedures merits a comprehensive review.

\section{GENERAL CONSIDERATIONS}

In general, airway-related complications remain a major cause of concern. The frequency and severity of many periprocedural complications vary depending on both patient and procedural factors. ERCP remains the most common advanced procedure, and its indications have expanded. The duration of many advanced procedures is variable, and the endoscopist's experience plays a crucial role. In this review, we mainly focus on procedures other than ERCP and EUS.

\section{ENDOBARIATRICS}

Endobariatric procedures may be employed as a primary 
treatment option or in patients who regained weight after a previous bariatric surgical procedure. In some respects, the challenges are similar, except that patients presenting for revisional procedures might have lost some weight. More important, patients who previously underwent weight loss surgery may also have long-term complications, such as severe acid reflux. Clearly, anesthesia providers should be aware of all pathophysiological changes associated with obesity and the resulting anatomical changes. These changes affect pharmacology, both in terms of dosing and clinical effects.

The procedures employed as primary weight loss options are primary obesity surgery endoluminal (POSE), ESG, insertion of intragastric balloons, aspiration therapy with the AspireAssist device, and gastric botulinum toxin injection for weight loss. The revisional bariatric procedure that is typically performed for endoluminal plication in patients who had undergone a Roux-en-Y gastric bypass is called transoral outlet reduction (TORe). The gastric pouch can also be decreased in size, which is called tubular TORe or restorative obesity surgery endoluminal (ROSE). Other techniques include mucosal ablation using argon plasma coagulation of the gastrojejunal anastomosis. ${ }^{1}$

POSE uses an incisionless operating platform to create full-thickness plications in the gastric fundus, thereby reducing gastric volume, which, in turn, limits the quantity of food that can be consumed at a given time. ${ }^{2}$ The procedure also causes antral dysmotility that prolongs satiety by inducing an earlier and longer feeling of gastric distention. In ESG, the endoscopist attempts to remodel the greater curvature of the stomach through the placement of full-thickness sutures. Similar to POSE, this results in reduced gastric capacity and delayed gastric emptying. ${ }^{3,4}$ Originally, ESG was performed using a superficial suction-based suturing device and had limited success owing to early suture loss. The current approach involves full-thickness suturing.

Both POSE and ESG are time-consuming procedures performed under general endotracheal anesthesia (GETA). In addition to the general risks associated with any endoscopic procedures, such as hypoxemia and aspiration, there are specific risks, including intraprocedural and postprocedural bleeding. Postprocedural pain could be substantial, requiring prolonged recovery room stay and occasional overnight hospitalization. There is an increased risk of suture release with continuous positive pressure application after the procedure. Pulmonary compliance can decrease as a result of abdominal and gastric distention due to carbon dioxide $\left(\mathrm{CO}_{2}\right)$ leakage, whereas both end-tidal $\mathrm{CO}_{2}\left(\mathrm{EtCO}_{2}\right)$ and pulmonary resistance may increase. Although the increase in $\mathrm{EtCO}_{2}$ can be addressed by increasing minute ventilation, needle insertion into the perito- neal cavity to release excess $\mathrm{CO}_{2}$ may be occasionally required for treating pneumoperitoneum.

Insertion of an intragastric balloon is typically performed under deep propofol sedation, more commonly referred to as monitored anesthesia care (MAC). These balloons cause weight loss by inducing gastroparesis. Depending on the type of balloon employed (either gas- or fluid-filled), they can be removed under MAC or GETA. Fluid-filled balloons should be removed under GETA because of the need to puncture the balloon to release the large volume of saline to facilitate balloon removal. All balloons should be removed, as they can migrate into the small bowel and cause obstruction.

The AspireAssist device (Aspire Bariatrics Inc., King of Prussia, PA, USA) functions similar to a percutaneous endoscopic gastrostomy tube and allows the aspiration of portions of ingested food from the stomach. It has been approved by the Food and Drug Administration for use in patients with a body mass index between 35 and $55 \mathrm{~kg} / \mathrm{m}^{2} .{ }^{2}$ Both the insertion and removal of this device are performed under deep sedation.

Gastric injection weight loss therapy involves injecting botulinum toxin into the smooth muscle of the gastric fundus to induce gastroparesis and early satiety. This is an effective weight-loss therapy in combination with diet and exercise. The procedure is performed under deep sedation.

Revisional endobariatric procedures such as TORe and ROSE utilize an endoscopic suturing device (Overstitch; Apollo Endosurgery, Austin, TX, USA) in patients who had undergone Roux-en-Y gastric bypass to narrow the gastrojejunal anastomosis and to decrease the size of the gastric pouch. These procedures are performed under GETA. Argon plasma coagulation involves the application of argon laser coagulation to the anastomotic outlet. Deep sedation is usually sufficient. Multiple sittings are needed, with each sitting taking approximately $15 \mathrm{~min}$.

Postoperative nausea and vomiting (PONV) can be a troubling complication of all endobariatric procedures. Aggressive pharmacotherapy has been used to limit PONV. Additionally, postprocedural pain may be substantial, particularly after full-thickness plication procedures. However, unlike bariatric surgery procedures, endoscopic procedures do not typically require hospital admission for monitoring, pain management, or nausea control.

At the Hospital of the University of Pennsylvania, our experience is with TORe, which is performed in our outpatient facility. Anesthesia is induced with propofol and fentanyl and maintained with infusions of propofol and remifentanil (an ultrashort-acting opioid). In morbidly obese patients, the doses of both propofol and remifentanil are lower than the standard adult doses (e.g., propofol bolus of $1.5-2 \mathrm{mg} / \mathrm{kg}$ body weight 
for induction). The infusions of both propofol and remifentanil are also considerably lower (approximately one-half) than those used for anesthesia maintenance in a normal-weight or overweight patient, approximately $70-100 \mu \mathrm{g} / \mathrm{kg} / \mathrm{min}$ propofol and $0.1-0.12 \mu \mathrm{g} / \mathrm{kg} / \mathrm{min}$ remifentanil. Blood pressure support often requires phenylephrine at approximately $30 \mu \mathrm{g} / \mathrm{min}$. Because of the increased risk of PONV, we avoid inhalational anesthetics. Intermittent positive pressure ventilation (IPPV) is always employed, and the settings are adjusted to maintain an acceptable $\mathrm{EtCO}_{2}$. The effect of rocuronium is reversed using sugammadex. Ondansetron is routinely administered, and dexamethasone is sometimes used for PONV prophylaxis. Postprocedural pain could be substantial, and patients are maintained in the recovery room for about 1 hour before being discharged home.

\section{POEM}

POEM is performed to treat achalasia, a motility disorder of the esophagus. Gastroenterologists often misdiagnose the condition as gastroesophageal reflux disease. The absence of relaxation of the lower esophageal sphincter is a characteristic feature of achalasia. Nevertheless, this is not essential for diagnosis and is not always present. ${ }^{5}$ The surgical treatment for this condition is a Heller myotomy, in which the anterior muscle fibers of the esophagus are incised longitudinally. ${ }^{6}$ Laparoscopic or thoracoscopic approaches, including laparoendoscopic single-site surgery and robot-assisted myotomy, and POEM, have largely replaced open surgery. ${ }^{7}$

The preprocedural evaluation of patients presenting for POEM should specifically focus on the increased risk of aspiration. The clinical presentation depends on the subtype of achalasia. Three subtypes are recognized: type I (classic), with minimal contractility in the esophageal body; type II, with intermittent periods of panesophageal pressurization; and type III (spastic), with premature or spastic distal esophageal contractions. Progressively worsening dysphagia initially to solids, followed by both solids and liquids, is the leading presentation. ${ }^{8}$ Severe reflux, history of regurgitation, pneumonia, and weight loss are signs of possible preprocedural/intraprocedural aspiration. Aspiration pneumonitis (acute and chronic), diffuse aspiration bronchiolitis, isolated bronchospasm, and aspiration pneumonia can occur in these patients. Chronic interstitial fibrosis is conceivable over a period of time and is related to periodic aspiration. ${ }^{9}$

In terms of anesthetic management, GETA is required for POEM. ${ }^{10}$ Extended fasting time is often required (up to $48 \mathrm{~h}$, depending on the degree of symptomatology, previous esoph- agogastroduodenoscopy [EGD] findings, and manometric studies) to minimize the risk of aspiration. Rapid sequence induction and intubation, often in a slight head-up position, has the least risk of aspiration. Even though suuden airway obstruction is rare, it can be caused by tracheomalacia, related to posterior tracheal cartilage ischemic damage resulting from chronic pressure due to massive esophageal dilation. The collapse is dynamic in nature, which can be relieved by IPPV with positive end-expiratory pressure. Cuffed reinforced endotracheal tubes are preferred to avoid kinking or obstruction of the endotracheal tube during endoscopy. In addition to standard monitoring, neuromuscular monitoring to ensure an appropriate degree of paralysis is essential. An increase in $\mathrm{EtCO}_{2}$ is anticipated and attributed to the use of $\mathrm{CO}_{2}$ by the endoscopist and its absorption. Any unexpected movement will not be appreciated by the endoscopist and should be avoided. The availability of sugammadex allows an anesthesia provider to err on the side of caution rather than risk inadequate paralysis. The procedure itself involves making a mucosal incision in the mid-esophagus, entering it, and creating a submucosal tunnel all the way to the gastric cardia by using a forward-viewing endoscope with a transparent distal cap and an ESD knife. Once the muscle fibers are exposed, they are incised and the mucosal incision is closed with endoscopic clips. ${ }^{11}$

Intraprocedural complications include pneumothorax, mediastinal emphysema, subcutaneous emphysema, pneumoperitoneum, and, rarely, capnopericardium. To diagnose these complications, a high degree of suspicion is necessary. ${ }^{12}$ The postprocedural period may be complicated by delayed hemorrhage, pleural effusion, minor inflammation or segmental atelectasis of the lungs, and gas under the diaphragm or aeroperitoneum.

Nearly $25 \%$ of patients undergoing POEM experience asymptomatic pneumothorax, especially when the procedure is performed under GETA. Positive pressure ventilation increases the air escape. Dissection of the thoracic portion of the esophagus and the resulting mediastinal pleural tear are the likely causes. Clearly, the use of air for insufflation must be avoided, which, despite potentially providing better distension and more manipulative space, is also associated with higher morbidity and mortality than the use of $\mathrm{CO}_{2}$.

Although a rare complication, capnopericardium can cause cardiac arrest. Disappearing $\mathrm{EtCO}_{2}$ tracing or pulse tracing on a plethysmogram may be the first indicators of capnopericardium. Blood pressure may be nonrecordable, and electrocardiogram changes might appear, including life-threatening arrhythmias. Immediate suspension of the procedure and endoscope withdrawal along with cardiopulmonary resuscitation may be required. Transthoracic echocardiography may 
not adequately image the heart. The procedure may need to be aborted, as any suturing of the mucosal incision might worsen the capnopericardium.

Subcutaneous emphysema is another known complication. There may be a palpable crepitus. $\mathrm{CO}_{2}$ insufflation may become challenging with both flow and pressure. An increase in $\mathrm{EtCO}_{2}$ may be noticed along with respiratory acidosis during blood gas measurement. Lung compliance will decrease (with increased airway pressure) along with sinus tachycardia and other cardiac arrhythmias. An increase in blood pressure is the likely result of an increase in partial $\mathrm{CO}_{2}>50 \mathrm{~mm} \mathrm{Hg}$. ${ }^{13}$ Decompression by inserting a 14- or 16-gauge angiocatheter in the right lower abdomen, approximately $5 \mathrm{~cm}$ below the costal margin, preferably guided by ultrasound, may become necessary. The presence of air in the mediastinum may not pose considerable clinical challenges and is typically observed along with subcutaneous emphysema. An increase in $\mathrm{EtCO}_{2}$, a decrease in saturation of peripheral oxygen $\left(\mathrm{SpO}_{2}\right)$, and inability to archive the desired tidal volume despite maximal safely attainable manual ventilation are key indicators of pneumomediastinum. The endoscope should be withdrawn, and the patient should be evaluated. Malignant hyperthermia should be excluded, although difficulty in ventilation and lack of hyperthermia can eliminate such a possibility. Acidosis will be seen on arterial blood gas, despite the administration of $100 \%$ oxygen. Hypotension, cardiac arrhythmia, and hemodynamic collapse are possible. Chest radiography might demonstrate variable pneumomediastinum and subcutaneous emphysema. De-aeration might become necessary and can be accomplished with a percutaneous abdominal needle along with positive pressure ventilation with suitable ventilator settings. ${ }^{14}$

All of the above complications may appear for the first time in the postprocedural period. At our hospital, patients presenting for POEM fast longer than usual and stay on clear fluids for 48 hours. We used to perform this procedure in our inpatient facility; however, after gaining more experience, we now perform about half of such procedures in our outpatient facility and the patients are usually discharged the next day. General anesthesia with propofol and fentanyl (generally 2-3 $\mathrm{mg} / \mathrm{kg}$ and 100-150 $\mu \mathrm{g}$, respectively), followed by intubation facilitated with either succinylcholine or rocuronium (usually $100 \mathrm{mg}$ for both) as a rapid sequence induction intubation technique, is used. Anesthesia is maintained with propofolremifentanil (infusion at $120-150 \mu \mathrm{g} / \mathrm{kg} / \mathrm{min}$ and $0.2-0.25 \mu \mathrm{g} /$ $\mathrm{kg} / \mathrm{min}$, titrated). $\mathrm{CO}_{2}$ accumulation is inevitable, and higher tidal volumes during IPPV are often needed. The incidence of PONV is higher than that with routine EGD, and prophylaxis with ondansetron is a standard practice. The procedure usually takes 1-2 hours.

\section{ESD}

ESD can be performed for en bloc resection of early GI luminal cancers. This procedure is performed on localized lesions with a minimal risk of metastasis. ${ }^{15,16}$ Unlike endoscopic mucosal resection, which can only remove lesions in a piecemeal fashion, ESD is intended to treat larger lesions with en bloc resection to assess the tumor-free margins. As a result, this procedure has a higher rate of complications, such as perforation causing peritonitis and delayed bleeding.

The endoscopist expects a relatively motionless and well-sedated patient. At our center, for lesions in the lower GI tract, ESD is generally performed under deep sedation with propofol. Occasionally, the procedure can take 2-3 hours. A short-acting opioid, such as fentanyl, is often required in increments and may contribute to longer recovery and postprocedural stays.

However, for upper GI lesions, GETA is preferred because of the risk of intraprocedural bleeding. It also ensures a stable working field without rapid or unexpected movements. It has been reported that the rate of curative resection is better and the perforation rate is lower in ESD performed under GETA than in ESD performed under conscious sedation after adjusting for several clinicopathologic factors, including the experience of the endoscopist, thereby improving oncologic outcomes in patients with superficial esophageal squamous cell carcinoma. ${ }^{16}$

At our hospital, general anesthesia with an endotracheal tube is always employed for ESD procedures. The induction doses of propofol and fentanyl are similar to those used in POEM; however, rapid sequence induction is not generally required. The maintenance of anesthesia is also similar. The procedure takes approximately 1-2 hours, and occasionally longer, depending on the size of the lesion. $\mathrm{CO}_{2}$ accumulation requires a higher tidal volume with IPPV. PONV prophylaxis with ondansetron is a standard practice. Postprocedural pain management may require an overnight stay.

\section{ERCP}

ERCP is a routine advanced procedure performed in many academic and freestanding endoscopy units. Although the issue of airway management is still unsettled, the choice is largely left to the institution and the individual anesthesia provider. Other reviews on this topic have extensively discussed the related issues. ${ }^{17,18}$

Cases of fatal and nonfatal air embolisms during ERCP have been reported. ${ }^{19-24}$ Other procedures such as EGD, EUS, colonoscopy, or sigmoidoscopy have also been associated with 
air embolism. ${ }^{25,26} \mathrm{~A}$ high index of clinical suspicion is required for diagnosis, and aggressive treatment is needed to avoid a fatal outcome. The use of precordial Doppler ultrasound and transesophageal echocardiography is helpful for establishing a diagnosis of air embolism. Besides traditional supportive measures, hyperbaric oxygen therapy should be considered in cases of suspected cerebral air embolism to improve neurological outcomes. Air embolism can also manifest for the first time after the procedure. A case of ventricular fibrillation requiring prolonged cardiopulmonary resuscitation and resulting in death in the recovery room has been reported. ${ }^{19}$ As previously stated, it is crucial that $\mathrm{CO}_{2}$ is used for advanced endoscopic procedures such as ERCP to decrease the risk of air embolism.

At our hospital, $>90 \%$ of ERCP procedures (including nearly $100 \%$ of procedures in the outpatient center) are performed under anesthesiologist (who is always a physician)-supervised deep propofol sedation administered by a certified nurse anesthetist. Propofol and fentanyl are typically administered as $80-100 \mathrm{mg}$ and $25-50 \mu \mathrm{g}$ boluses, respectively, followed by the infusion of $80-120 \mu \mathrm{g} / \mathrm{kg} / \mathrm{min}$ propofol. The patients breathe spontaneously, and supplemental oxygen is generally administered through a nasal trumpet introduced into the nose or mouth (Fig. 1).

\section{EUS-GUIDED DRAINAGE OF PERIPANCREATIC COLLECTIONS}

EUS-guided drainage of large pancreatic pseudocysts and pancreatic walled-off necrosis to allow for transgastric necrosectomy are rare procedures. These procedures are performed in patients with pancreatitis and typically in an inpatient facility, although they can also be performed in an outpatient facility.

Ultrasound-guided drainage of pancreatic cysts, including pseudocysts, may be performed under deep sedation if the cysts are small and have a negligible risk of pulmonary aspiration resulting from the leak/flooding of the stomach. However, most pseudocysts that require drainage are large and contain a large volume of fluid that will be released into the GI lumen. As there is a risk of pulmonary aspiration with large cysts, GETA tube is advisable.

Patients with infected peripancreatic walled-off necrosis collections will also require drainage and multiple sessions of direct endoscopic necrosectomy. Patients with severe necrotizing pancreatitis can develop a well-demarcated, organized collection of necrotic tissue. ${ }^{27} \mathrm{~A}$ transgastric stent can be placed into the collection to facilitate direct endoscopic necrosectomy for the endoscopic debridement of this collection. This approach is known to result in a lower incidence of new-onset multiorgan failure and a lower major complication rate than surgical necrosectomy. In contrast to open surgical necrosectomy, direct endoscopic necrosectomy is associated with lower rates of complications such as bleeding and inadvertent puncture of the adjacent viscera, as well as with a decreased risk of chronic pancreaticocutaneous fistulae. This procedure is always performed under GETA.

We recognize that the anesthesia/sedation practice for advanced procedures, especially ERCP, varies worldwide. In most countries (outside the United States), the majority of ERCP and EUS procedures are performed either with intravenous conscious sedation or a registered nurse (not a nurse anesthetist)-administered propofol sedation. Generally, the propofol doses are lower. As a likely result, the satisfaction of both endoscopists and patients is lower. Nonetheless, the prac-

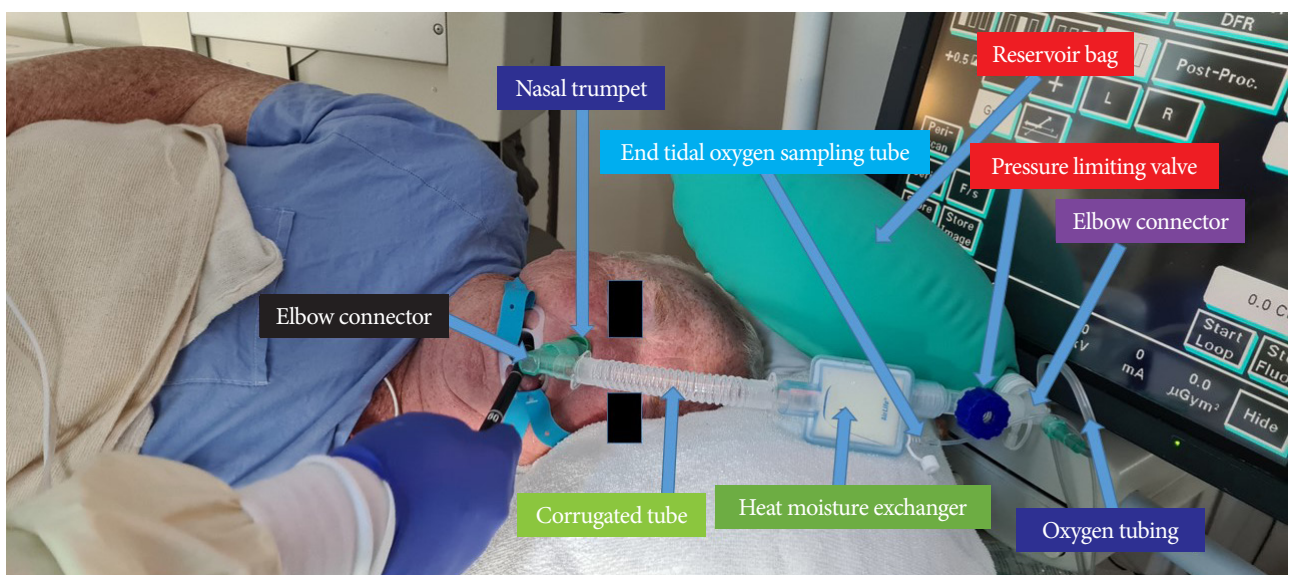

Fig. 1. Mapleson $C$ breathing system in use in a obese patient undergoing esophageal dilation with propofol deep sedation. 


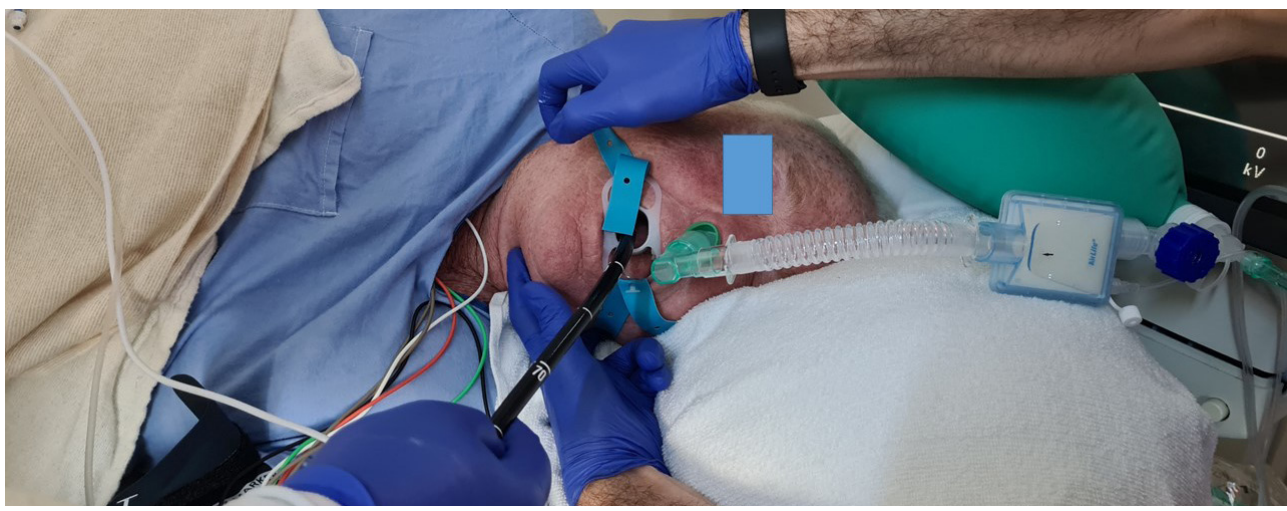

Fig. 2. Head tilt-chin lift and jaw-thrust maneuvers in use to restore upper airway patency.

tice is safe and, in fact, a meta-analysis has shown it to be safer than physician anesthesiologist-administered propofol sedation. ${ }^{28}$ For the advanced procedures discussed here, with the exception of uncomplicated ERCP and EUS, we recommend that the sedation be administered or supervised by an anesthesiologist.

Irrespective of the person administering the sedation, adverse events such as desaturation are inevitable. The most common causes of desaturation are hypoventilation (as a result of sedative medications) and upper airway obstruction. An attentive clinician/nurse will be able to recognize the cause. The corrective measures depend on the cause. Airway obstruction often responds to measures such as chin lift, jaw thrust, and neck extension - maneuvers often not employed or employed late (Fig. 2). The oxygen flow should be immediately increased to $10-15 \mathrm{~L} / \mathrm{min}$. If these measures are ineffective, the endoscopist must withdraw the endoscope, and bag mask ventilation should begin in earnest. If laryngospasm is recognized, the short-acting muscle relaxant succinylcholine should be administered at approximately $25-50 \mathrm{mg}$, early than late. Certain factors increase the risk of hypoventilation and airway obstruction. Elderly patients are particularly susceptible to hypoventilation, even with small doses of sedative medications. Smokers, obese individuals, those with obstructive sleep apnea, patients with chronic obstructive pulmonary disease, and those recovering from a recent upper respiratory infection are susceptible to upper airway obstruction, whereas patients with asthma are predisposed to bronchial obstruction.

\section{CONCLUSIONS}

In conclusion, advanced GI endoscopic procedures continue to evolve and pose many unique challenges to the anesthe- sia provider. Endoscopists are also likely to be in a "learning mode" during the early stages of the introduction of these procedures in their procedure settings. A discussion between the anesthesia provider and the endoscopist is crucial to recognize the risks and preempt them. As always, the team should be prepared to manage both expected and unexpected adverse events.

\section{Conflicts of Interest}

The authors have no potential conflicts of interest.

Funding

None.

Author Contributions

Conceptualization: Basavana Goudra, Monica Saumoy

Data curation: BG, MS

Writing-original draft: BG, MS

Writing-review\&editing: BG, MS

$\begin{array}{ll}\text { ORCID } & \\ \text { Basavana Goudra } & \text { https://orcid.org/0000-0002-5146-0424 } \\ \text { Monica Saumoy } & \text { https://orcid.org/0000-0003-2780-1206 }\end{array}$

\section{REFERENCES}

1. Goh YM, James NE, Goh EL, Khanna A. The use of endoluminal techniques in the revision of primary bariatric surgery procedures: a systematic review. Surg Endosc 2020;34:2410-2428.

2. Tawadros A, Makar M, Kahaleh M, Sarkar A. Overview of bariatric and metabolic endoscopy interventions. Ther Adv Gastrointest Endosc 2020;13:2631774520935239.

3. de Moura DTH, de Moura EGH, Thompson CC. Endoscopic sleeve gastroplasty: from whence we came and where we are going. World J Gastrointest Endosc 2019;11:322-328.

4. Jain D, Bhandari BS, Arora A, Singhal S. Endoscopic sleeve gastroplasty - a new tool to manage obesity. Clin Endosc 2017;50:552-561. 
5. Fox M, Hebbard G, Janiak P, et al. High-resolution manometry predicts the success of oesophageal bolus transport and identifies clinically important abnormalities not detected by conventional manometry. Neurogastroenterol Motil 2004;16:533-542.

6. Torres-Villalobos G, Martin-Del-Campo LA. Surgical treatment for achalasia of the esophagus: laparoscopic Heller myotomy. Gastroenterol Res Pract 2013;2013:708327.

7. Goudra B, Singh P, Green M. Anaesthesia for uncommon and emerging procedures. New York (NY): Springer; 2021.

8. Patel DA, Lappas BM, Vaezi MF. An overview of achalasia and its subtypes. Gastroenterol Hepatol (NY) 2017;13:411-421.

9. Ali HA, Murali G, Mukhtar B. Respiratory failure due to achalasia cardia. Respir Med CME 2009;2:40-43.

10. Goudra B, Singh PM, Gouda G, Sinha AC. Peroral endoscopic myotomy-initial experience with anesthetic management of 24 procedures and systematic review. Anesth Essays Res 2016;10:297-300.

11. Pandolfino JE, Kahrilas PJ. Presentation, diagnosis, and management of achalasia. Clin Gastroenterol Hepatol 2013;11:887-897.

12. Ren Z, Zhong Y, Zhou P, et al. Perioperative management and treatment for complications during and after peroral endoscopic myotomy (POEM) for esophageal achalasia (EA) (data from 119 cases). Surg Endosc 2012;26:3267-3272.

13. Bang Y-S, Park C. Anesthetic consideration for peroral endoscopic myotomy. Clin Endosc 2019;52:549-555.

14. Okada T, Izuta S, Mizobuchi S. A case of ventilatory impairment during per-oral endoscopic myotomy under general anesthesia. JA Clin Rep 2018;4:23.

15. Kiriyama S, Naitoh H, Kuwano H. Propofol sedation during endoscopic treatment for early gastric cancer compared to midazolam. World J Gastroenterol 2014;20:11985-11990.

16. Song BG, Min YW, Cha RR, et al. Endoscopic submucosal dissection under general anesthesia for superficial esophageal squamous cell carcinoma is associated with better clinical outcomes. BMC Gastroenterol 2018;18:80.

17. Goudra B, Singh PM. ERCP: the unresolved question of endotracheal intubation. Dig Dis Sci 2014;59:513-519.
18. Basavana G. Goudra, Michael Duggan, Vidya Chidambaran, et al. Anesthesiology: a practical approach. New York (NY): Springer; 2018.

19. Hauser G, Milosevic M, Zelić M, Stimac D. Sudden death after endoscopic retrograde cholangiopancreatography (ERCP)--case report and literature review. Medicine (Baltimore) 2014;93:e235.

20. Wanderer JP, Nathan N. Bubble trouble: venous air embolism in endoscopic retrograde cholangiopancreatography. Anesth Analg 2018;127:324.

21. Marchesi M, Battistini A, Pellegrinelli M, Gentile G, Zoja R. Fatal air embolism during endoscopic retrograde cholangiopancreatography (ERCP): an "impossible" diagnosis for the forensic pathologist. Med Sci Law 2016;56:70-73.

22. Mathew J, Parker C, Wang J. Pulseless electrical activity arrest due to air embolism during endoscopic retrograde cholangiopancreatography: a case report and review of the literature. BMJ Open Gastroenterol 2015;2:e000046.

23. Wills-Sanin B, Cárdenas YR, Polanco L, Rivero O, Suarez S, Buitrago AF. Air embolism after endoscopic retrograde cholangiopancreatography in a patient with Budd Chiari syndrome. Case Rep Crit Care 2014;2014:205081.

24. Lanke G, Adler DG. Gas embolism during endoscopic retrograde cholangiopancreatography: diagnosis and management. Ann Gastroenterol 2019;32:156-167

25. Fang Y, Wu J, Wang F, Cheng L, Lu Y, Cao X. Air embolism during upper endoscopy: a case report. Clin Endosc 2019;52:365-368.

26. Donepudi S, Chavalitdhamrong D, Pu L, Draganov PV. Air embolism complicating gastrointestinal endoscopy: a systematic review. World J Gastrointest Endosc 2013;5:359-365.

27. Thompson CC, Kumar N, Slattery J, et al. A standardized method for endoscopic necrosectomy improves complication and mortality rates. Pancreatology 2016;16:66-72.

28. Goudra BG, Singh PM, Gouda G, et al. Safety of non-anesthesia provider-administered propofol (NAAP) sedation in advanced gastrointestinal endoscopic procedures: comparative meta-analysis of pooled results. Dig Dis Sci 2015;60:2612-2627. 